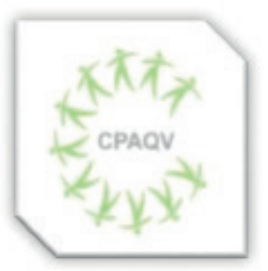

ISSN: $2178-7514$
ARTIGO DE REVISÃO

\section{BIOMECÂNICA APLICADA AO DESEMPENHO FÍSICO E PREVENÇÃO DE LESÕES EM JOGADORES DE FUTEBOL}

Vol. 13| No.1| Ano 2021

Taís Rocha dos Santos ${ }^{1}$; Bráulio Nascimento Lima ${ }^{2,7}$; Vanessa Borges Gomes ${ }^{2}$; Thales Henriques Pires Cruz ${ }^{3}$; José Ricardo Lourenço Oliveira ${ }^{5,7}$; Leandro Borelli de Camargo ${ }^{5,7}$; Carlos Henrique Prevital Fileni ${ }^{5,7}$; Luís Augusto da Silva Nogueira Filho ${ }^{4}$; Valeria de Albuquerque Pires ${ }^{4}$; Klebson da Silva Almeida,7; Ricardo Pablo Passos ${ }^{5,6,7}$; Lilia Jarina Almeida Martins dos Santos ${ }^{8}$; Guanis de Barros Vilela Junior ${ }^{5,7}$; Luís Felipe Sílio ${ }^{1,7}$

\title{
RESUMO
}

O futebol é o esporte mais praticado no Brasil, sendo que ocorrem campeonatos em todo o mundo, nos quais se espera por resultados satisfatórios das equipes o que leva os grandes clubes a investirem em infraestrutura e na busca por profissionais qualificados que possam colaborar para a eficiência no rendimento e desempenho dos atletas. Neste contexto a biomecânica vem sendo aplicada no desempenho físico de muitos atletas. Questiona-se assim, como a análise da biomecânica aplicada ao desempenho físico na modalidade do futebol, auxilia a evitar lesões. Baseado nisso, a presente pesquisa tem por objetivo apresentar uma revisão bibliográfica sobre a aplicabilidade da biomecânica para evitar lesões, em função do aprimoramento do desempenho físico na modalidade do futebol. Para tanto foi realizada uma revisão bibliográfica com levantamento de artigos científicos nas seguintes bases de dados: Literatura Latino-Americana e do Caribe em Ciências da Saúde (LILACS), Literatura Internacional em Ciências da Saúde (PUBMED), na Biblioteca Eletrônica Científica Online (Scielo) e Google Scholar o que possibilitou a busca por estudos publicados no cenário nacional e internacional, com corte temporal de 2017 a 2020, que contemplam os objetivos da pesquisa, assim como também as pesquisas indexadas e disponíveis na integra para leitura e análise. No levantamento do material, foram incluídos 9 artigos para análise e discussão. Para apresentação dos resultados foi utilizado quadro demonstrativo apresentando os objetivos e os métodos utilizados em cada estudo. Pode-se concluir que as investigações biomecânicas nos atletas de futebol contribuem significativamente para o prolongamento da carreira esportiva, evitando a interrupção por afecções crônico-degenerativas, padronizando procedimentos para a analisar o movimento esportivo de forma mais precisa e científica.

Palavras-chave: Biomecânica; Futebol; Lesões Esportivas.

\section{ABSTRACT}

Football is the most practiced sport in Brazil, with championships taking place all over the world, in which teams are expected to achieve satisfactory results, which leads big clubs to invest in infrastructure and in the search for qualified professionals who can collaborate to efficiency in performance and performance of athletes. In this context, biomechanics has been applied to the physical performance of many athletes. Thus, it is questioned how the analysis of biomechanics applied to physical performance in football modality helps to avoid injuries. Based on this, the present research aims to present a bibliographic review on the applicability of biomechanics to prevent injuries, due to the improvement of physical performance in football. To this end, a bibliographic review was carried out with a survey of scientific articles in the following databases: Latin American and Caribbean Literature in Health Sciences (LILACS), International Literature in Health Sciences (PUBMED), in the Online Electronic Scientific Library (Scielo) ) and Google Scholar, which made it possible to search for studies published in the national and international scenario, with a time cut from 2017 to 2020, which contemplate the research objectives, as well as the indexed and available researches for reading and analysis. In the survey of the material, 9 articles were included for analysis and discussion. To present the results, a demonstrative table was used showing the objectives and methods used in each study. It can be concluded that the biomechanical investigations in soccer athletes contribute significantly to the prolongation of the sports career, avoiding interruption due to chronic-degenerative conditions, standardizing procedures to analyze the sports movement in a precise and scientific way.

Keywords: Biomechanics; Football; Sports Injuries.

Autor de correspondência

Luís Felipe Sílio - silioeducafisica@gmail.com
1Centro Universitário São Lucas - RO

2Faculdade Conhecimento e Ciência (FCC) - PA

3Instituto Federal do Pará (IFPA) - PA

4Universidade da Amazônia (UNAMA) - PA

5Universidade Metodista de Piracicaba (UNIMEP) - SP

6Faculdade Anhanguera de Sumaré - SP

7Núcleo de Pesquisas em Biomecânica Ocupacional e Qualidade de Vida (NPBOQV)

8 Fundação Universidade Federal de Rondônia 


\section{INTRODUÇÃO}

O futebol é o esporte mais popular do mundo, com aproximadamente 265 milhões de jogadores em todo o mundo(1). No Brasil, o futebol começou a ser praticado no final do século XIX e desde então se popularizou e também é o esporte mais praticado no país, que atrai $42,7 \%$ dos praticantes de esporte. Esse número expressivo de praticantes de futebol no Brasil pode ser reflexo do próprio histórico esportivo no país, considerando sobretudo as razões históricas e culturais, e a forte influência da mídia(2).

O futebol por ser um esporte com bastante movimentação, é uma modalidade que provoca várias lesões sendo as mesmas de ordem ortopédica, muscular, ligamentar e tendinosas ${ }^{(3)}$. Neste sentido, Cristi-Sánchez et al. ${ }^{(1)}$, afirmam que as lesões no futebol são comuns, com uma taxa de 2,6 lesões por jogo sendo relatadas durante torneios internacionais de elite. Particularmente, lesões por uso excessivo são um grande problema em futebol, responsável por quase um terço da incidência de lesões.

Nesse sentindo, os principais fatores de riscos associados a lesões no futebol são o excesso de treino, a falta de descanso e a recuperação inadequada ${ }^{(3)}$.

As causas das lesões podem ser definidas como multifatoriais, sendo considerados fatores intrínsecos como instabilidade articular, força muscular, tensão muscular, assimetrias, condições biomecânicas e psicológica ${ }^{(4)}$.

O estudo desenvolvido por Silva et $\mathrm{al}^{(3)}$, apontam como lesões mais incidentes, provenientes do futebol, o estiramento muscular, sendo os membros inferiores os mais agravantes e o jogadores que jogam na posição de meio campo como os que mais sofrem lesões.

Também foi observado no estudo, os principais fatores de riscos associados a lesões no futebol: o excesso de treino, a falta de descanso e a recuperação inadequada como principais fatores de riscos ${ }^{(3)}$.

Desta forma, estudos investigativos são empregados com base na biomecânica com o intuito de prevenir ou reduzir as lesões no futebol $^{(5)}$.

Silva, Batista e $\operatorname{Keller}^{(6)}$, explicam que a Biomecânica é uma das áreas da ciência que tem ferramentas para investigar quantitativamente o futebol.

Para que se compreenda melhor de que forma a análise da biomecânica aplicada ao desempenho físico auxilia na modalidade do futebol, temos como parâmetro os métodos de medição utilizados: a cinemática, a eletromiografia, a dinamometria e a antropometria ${ }^{(6)}$.

Dufour e Pillu(5), afirmam que os referidos métodos são utilizáveis justificando que o movimento pode ser descrito e modelado matematicamente, permitindo a maior compreensão dos mecanismos internos reguladores e executores do movimento do corpo humano. 
A medição cinemática é um método que busca, por meio da aquisição de imagens da execução do movimento, analisar o comportamento de variáveis dependentes, como a velocidade, o deslocamento, a posição e a orientação do corpo e de suas partes, de forma a fornecer dados para uma análise biomecânica quantitativa dos movimentos humanos, pois, atualmente, ela é muito utilizada para estudar os fundamentos, os sistemas de jogo e as capacidades físicas, entre outros ${ }^{(6)}$.

Outro método amplamente utilizado, a dinamometria isocinética engloba todos os tipos de medidas de força de forma a poder interpretar as respostas de comportamentos dinâmicos do movimento humano ${ }^{(7)}$.

Ademais, a eletromiografia é o método que registra a atividade elétrica do músculo quando esse realiza contração. A EMG é também considerada uma área importante de avaliação biomecânica, por ser um meio de que dispomos atualmente para medir, ainda que indiretamente, as forças produzidas pelos grupos musculares, entrando assim, no âmbito da Biomecânica interna ${ }^{(8)}$.

A antropometria procura determinar características e propriedades do aparelho locomotor como as dimensões das formas geométricas de segmentos corporais, distribuição de massa, braços de alavanca, posições articulares etc., definindo um modelo antropométrico a partir das medidas corporais ${ }^{(5)}$
Assim, problematiza-se neste estudo como a análise da biomecânica aplicada ao desempenho físico na modalidade do futebol, auxilia a evitar lesões. Para que possa se obter respostas acerca da problematização apresentada, será feita a análise de revisão da literatura de cunho exploratória e descritiva, de abordagem quantitativa que tratam sobre a temática. Com abordagem para os temas pertinentes: Biomecânica, Futebol, Lesões Esportivas.

Verifica-se o grande número de pessoas adeptas do futebol no Brasil e no mundo com a finalidade de praticar esportes e lazer. Porém, com o aumento da prática do futebol, há também o risco eminente de lesões associadas ao futebol sendo necessário maiores investigações sobre como prevenir tais lesões e como a biomecânica se insere nesse contexto, visto que através do seu emprego, pode-se estudar as técnicas do movimento e otimizar a condição física dos atletas, visto que pode desenvolver ferramentas para diagnóstico das lesões mais comuns, por exemplo $^{(3)}$

Diante da relevância do tema, faz-se importante verificar o que se tem relatado na literatura científica sobre como a análise da biomecânica aplicada ao desempenho físico na modalidade do futebol, auxilia a evitar lesões.

Portanto, o objetivo primário deste trabalho é apresentar uma revisão bibliográfica sobre a aplicabilidade da biomecânica para evitar lesões, em função do aprimoramento do desempenho físico na modalidade do futebol. 


\section{MÉTODOS}

A fim de alcançar os objetivos utilizou-se a revisão bibliográfica de abordagem qualitativa. Primeiramente se identificou o tema e seleção da hipótese ou questão de pesquisa para a elaboração da revisão bibliográfica.

Como critério de inclusão, foram selecionados artigos científicos em forma de documentos eletrônicos de bancos de dados científicos na busca e alocação de conhecimento sobre a biomecânica aplicada ao desempenho físico como forma de auxiliar a evitar lesões. Os arquivos selecionados devem apresentar dados que forneçam valores sobre métodos usados na biomecânica, correlacionando tal conhecimento com as abordagens e publicados entre os anos de 2017 à 2020.

O presente estudo busca resposta, para o problema de pesquisa: Como a análise da biomecânica aplicada ao desempenho físico na modalidade do futebol, auxilia a evitar lesões?

As palavras-chave utilizadas na linha de busca dos bancos de dados científicos foram:
Biomecânica, Futebol, Lesões Esportivas.

O levantamento bibliográfico foi realizado nas bases de dados da Literatura LatinoAmericana e do Caribe em Ciências da Saúde (LILACS), Literatura Internacional em Ciências da Saúde (PUBMED), na Biblioteca Eletrônica Científica Online (Scielo) e Google Scholar o que possibilitou a busca por estudos publicados no cenário nacional e internacional, com corte temporal de 2017 a 2020.

\section{RESULTADOS}

Foram encontrados 48 artigos nas bases de dados, dos quais, em um primeiro momento, foram excluídos 08 estudos por estarem indisponíveis, 07 por estarem inferiores ao período previamente selecionado, 08 por estarem duplicados, 06 por serem artigos de revisão, 09 por serem teses, sobrando apenas 10 artigos para leitura na íntegra. Após leitura na integra foram incluídos 9 estudos que passaram a compor o presente estudo. O fluxograma do processo de filtragem encontra-se na figura 01 .

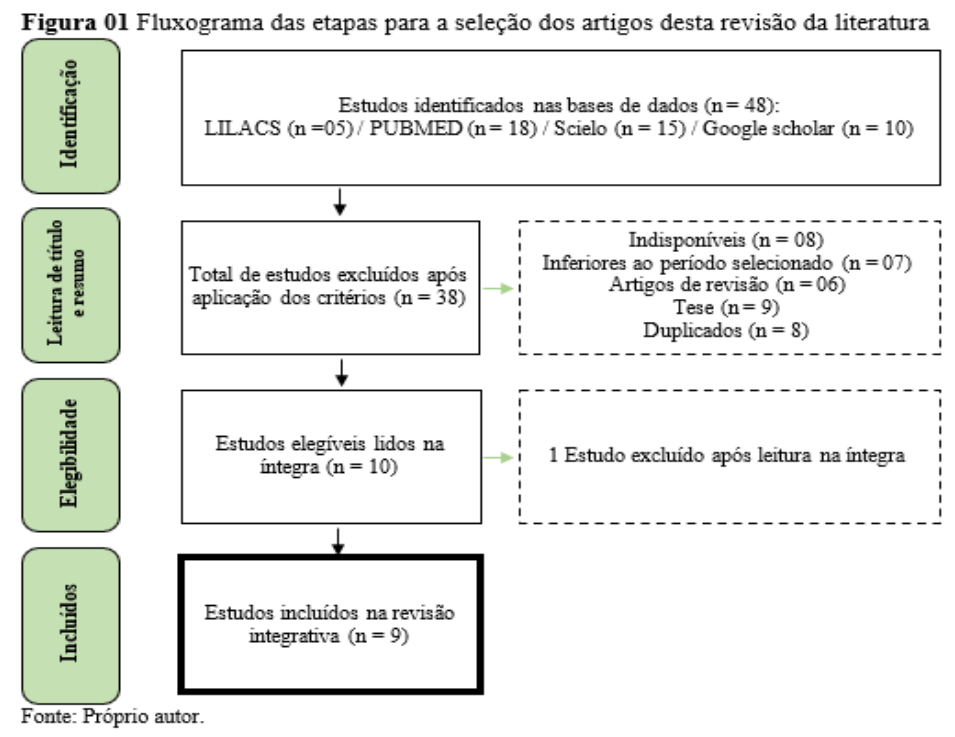


A seguir, pode-se verificar na tabela 01 biomecânica aplicada ao desempenho físico na que a literatura científica levantada, num total modalidade do futebol, auxilia a evitar lesões. de 09 artigos mostrou o entendimento sobre

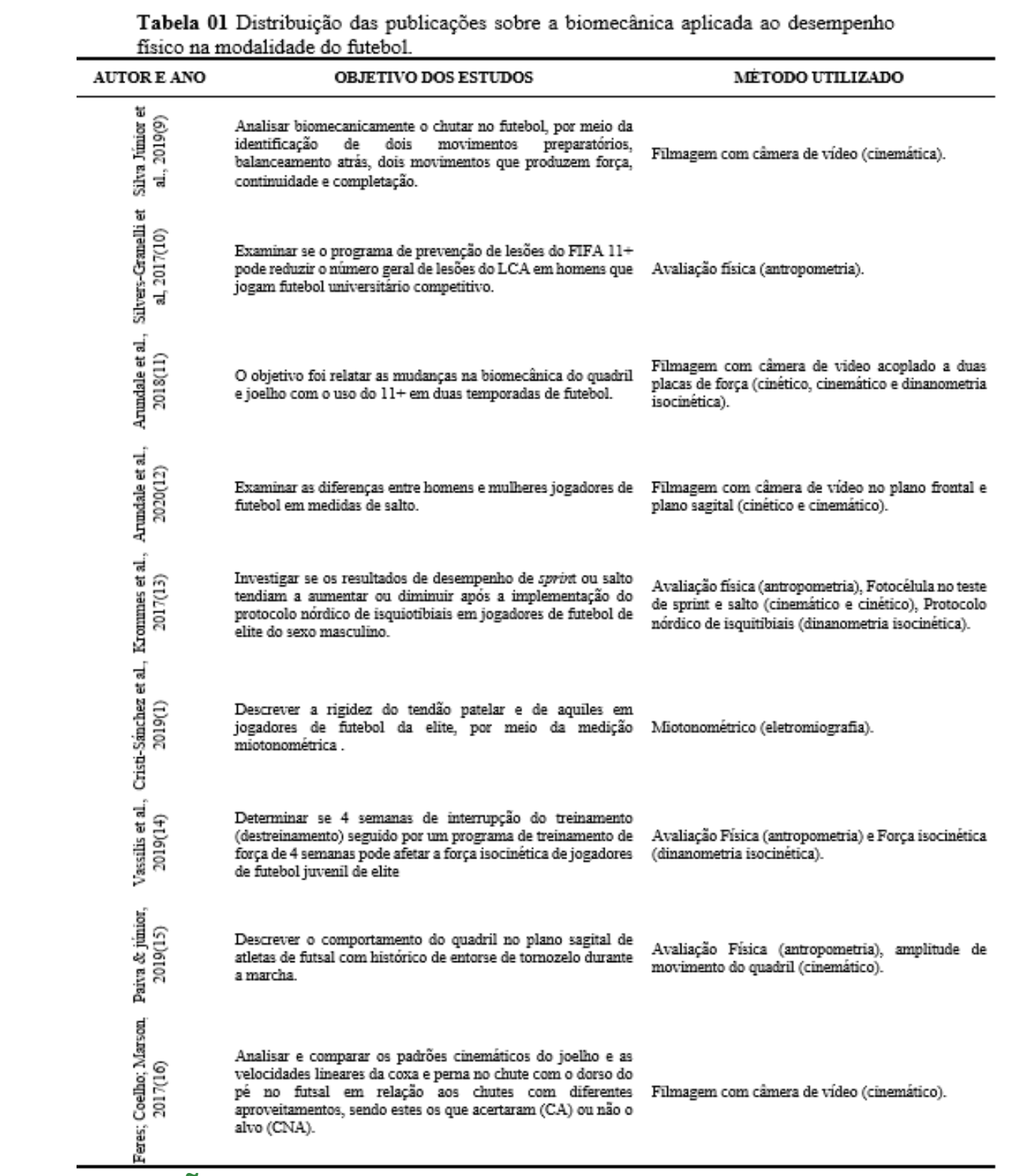

\section{DISCUSSÃO}

Diante da literatura consultada, como se pode ver no quadro 1, foram encontradas 9 publicações (entre os anos de 2017 a 2019) que contemplam a importância dos métodos cinemáticos, antropométricos, dinanométricos e eletromiográficos para a prevenção e melhora no desempenho dos jogadores de futebol.

Os estudos encontrados são fundamentados na biomecânica, visto que vem se destacando cada vez mais por seu emprego na otimização do rendimento esportivo, inclusive do futebol de várias maneiras: realizando a análise 
da técnica de movimento e da condição física dos atletas com o intuito de reduzir sobrecargas excessivas $^{(16)}$.

Desta forma apresentam-se os resultados de 09 estudos que mostram como a biomecânica aplicada ao esporte consegue colaborar para progredir, modernizar, automatizar e prevenir lesões e outros acometimentos maléficos aos atletas de futebol, padronizando procedimentos para a analisar o movimento esportivo de forma precisa e científica ${ }^{(5)}$.

O estudo de Silva Júnior et al., ${ }^{(3)}$, buscou analisar biomecanicamente o chute no futebol, por meio da identificação de dois movimentos preparatórios, balanceamento atrás, dois movimentos que produzem força, continuidade e completação. Participou do estudo um atleta profissional de futebol que realizou um arremesso tentando acertar ou acertar um gol com a maior velocidade possível da bola, sendo filmado em plano não sagital por uma câmera digital a 30 Hz. Como imagens do tiro foram analisadas no software Kinovea. Os resultados demonstram que uma análise biomecânica é fundamental para verificar e corrigir falhas em movimentos que podem estar diretamente relacionadas ao modo como os atletas chutam, buscando também otimizar ou melhorar o movimento.

Silvers-Granelli e colaboradores (10), elaboraram um estudo com o propósito de examinar se o programa de prevenção de lesões do FIFA $11+$ pode reduzir o número geral de lesões do LCA em homens que jogam futebol universitário competitivo. O referido programa demonstrou diminuir o risco de lesões no futebol em homens e mulheres. O programa também demonstrou diminuir a perda de tempo resultante de lesões. No entanto, estudos anteriores não investigaram especificamente como o programa pode impactar a taxa de lesão do ligamento cruzado anterior (LCA) em jogadores de futebol do sexo masculino. Este programa, se implementado corretamente, tem o potencial de diminuir a taxa de lesão do LCA em jogadores de futebol de competição. Além disso, isso também pode aprimorar o desenvolvimento e a disseminação de protocolos de prevenção de lesões e pode reduzir o risco para os atletas que utilizam o programa de forma consistente.

Estudo de Arundale et al., ${ }^{(11)}$ colabora com Silvers- Granelli ${ }^{(10)}$ sobre ocorrer mudanças na biomecânica do quadril e joelho com o uso do $11+$ em duas temporadas de futebol. Todavia, em outro estudo, no qual dois times universitários de futebol feminino realizaram o $11+$ em duas temporadas de futebol. Uma equipe de controle foi seguida por uma temporada. Os atletas realizaram análises de movimento de um salto vertical de queda durante a pré-temporada e pós-temporada. Ambos os grupos tiveram aumento significativos no pico do ângulo de abdução do joelho durante a primeira temporada, e não houve mudanças significativas no pico do momento de abdução do joelho em qualquer uma das temporadas. O grupo controle teve diminuições bilaterais no ângulo de flexão do joelho. O programa não 
pareceu impactar sistematicamente os fatores de risco biomecânicos associados a lesões no joelho, com aumento no ângulo de abdução de pico do joelho sem alterações bilaterais no movimento frontal ou transversal do quadril. O $11+$ pode ter atenuado reduções clinicamente significativas na flexão do joelho, no entanto, como as lesões do LCA não ocorrem puramente no plano sagital, não está claro o impacto dessas alterações.

Dentro da periodização nos programas de treinamento voltado para o futebol, as fases de treinamento especifícos e fases de destreinamento são essenciais para prevenções de sobrecarga no atleta.

Vassilis et al.(14), elaboraram um estudo com objetivo de determinar se 4 semanas de interrupção do treinamento (destreinamento) seguido por um programa de treinamento de força de 4 semanas pode afetar a força isocinética de jogadores de futebol juvenil de elite. Participaram do estudo 13 jogadores. Os jogadores realizaram medidas antropométricas e medidas isocinéticas de força de membros inferiores 3 vezes, antes da interrupção do treinamento, após a interrupção do treinamento e após o programa de treinamento de força de 4 semanas. Não foram observadas diferenças significativas nas medidas antropométricas e de força $(\mathrm{P}>0,05)$ após o período de destreinamento e após o programa de treinamento $(\mathrm{P}>0,05)$. Esses resultados indicam que 4 semanas de destreinamento em jogadores de futebol juvenil de elite não tem efeitos significativos de acordo com suas características antropométricas e força isocinética de seus membros inferiores. Além disso, nem o programa de treinamento de 4 semanas afetou os parâmetros acima. Talvez os jovens jogadores consigam manter os benefícios do treinamento melhor do que os adultos devido às suas adaptações neurais. A duração do programa de treinamento de força pode ser o motivo da falta de adaptações.

Nos movimentos básicos e avançados na dinâmica da modalidade futebol, a articulação do quadril é amplamente utilizada e exerce grande demanda dos atletas, principalmente nos movimentos técnicos.

O estudo de Paiva e Soares Júnior ${ }^{(15)}$, se concentrou no objetivo de descrever o comportamento do quadril no plano sagital de atletas de futsal com histórico de entorse de tornozelo durante a marcha, assim, as informações coletadas através da análise laboratorial foram descritas de forma direta e tendo como referência de normalidade, dados de indivíduos controle normais e valores da literatura. Juntamente com a velocidade angular. A dinâmica da articulação do quadril estudado durante a marcha, nos mostrou alterações da cinemática do movimento no plano sagital especialmente valores da amplitude de movimento. Pelo fato do presente estudo ter tido uma amostra convencional e ser de caráter descritivo, consideraram-se os dados encontrados uma descoberta inicial e potencial para futuros estudos, que possam englobar amostras maiores e a apresentação dos distúrbios biomecânicos do 
atleta de futsal em ambos os membros inferiores frente a esse tipo de lesão, somados a possíveis causas e disfunções nos complexos articulares e musculares, como por exemplo, a utilização da análise eletromiográfica dos músculos envolvidos na marcha e até mesmos nos gestos do esporte.

O estudo e Arundale et. al. ${ }^{(12)}$, com base na biomecânica, com o intuito de examinar as diferenças entre homens e mulheres jogadores de futebol na cinética do salto, para isso, foram combinados com base na idade, frequência de treinamento e posição de jogo. Todos os jogadores realizaram o tuck jump (salto vertical no solo) e drop jump vertical (salto vertical com saída de uma plataforma alta, seguido de um salto no solo). Os resultados indicam que as mulheres têm pior desempenho na avaliação do tuck jump do que os homens. Os resultados apoiam descobertas anteriores de que as mulheres apresentam maior mediação dos joelhos (valgo dinâmico) do que os homens, mas também descobriram que os homens podem ter maiores assimetrias em joelhos valgos. Estes resultados de medidas cinéticas fornecem algumas sugestões para os médicos considerarem durante a reabilitação da reconstrução do LCA para melhora dos desempenhos dos atletas.

A musculatura posterior de coxa (isquiotibiais) está entre os grupamentos musculares mais utilizados nos gestos motores do futebol, tendo em vista o alto nível de lesão durante as temporadas de elite.

O estudo de Krommes et al. ${ }^{(13)}$, aplicou o Protocolo Nordic Hamstring -NHP (Protocolo
Nórdico dos Isquiotibiais) em nível de equipe, a fim de monitorar seu efeito no desempenho de sprint e salto em jogadores de futebol masculino de elite e reduzir as lesões por distensão dos isquiotibiais em jogadores de futebol. Os autores investigaram se os resultados de desempenho de sprint ou salto tendiam a aumentar ou diminuir após a implementação do método NHP. Podese considerar que realizar o NHP em jogadores de elite, não pareceu afetar negativamente os resultados de desempenho de sprint e salto vertical no presente estudo, embora na verdade tenha mostrado uma significativa melhora para as características mais explosivas, como os tempos parciais de 5 e 10 metros curtos e altura máxima do salto, que são parâmetros de desempenho altamente relevantes no futebol de elite.

A fim de otimizar a reabilitação em estágios patológicos da rigidez do tendão por medição miotonométrica (uma técnica não invasiva e indolor baseada na teoria da oscilação livre, onde um breve impulso mecânico é realizado sobre a pele usando uma sonda e parâmetros), 49 atletas de futebol de elite foram avaliados. Um dispositivo portátil foi usado para medir a rigidez do tendão de Aquiles e patelar. membros dominantes e não dominantes foram avaliados em ambos os grupos. Os autores afirmaram que a maior rigidez do tendão patelar pode estar relacionada a uma melhora na transmissão de força durante a contração muscular. Por outro lado, parece que após anos de treinamento profissional, a rigidez do tendão de Aquiles não 
muda, conservando a função de armazenamentoliberação de energia elástica. As diferenças não significativas entre as posições podem ser atribuídas aos anos de treinamento homogêneo que os jogadores realizaram. Verificou-se que a rigidez do tendão, a relação entre a força aplicada a um tendão e o deslocamento exercido, pode ajudar a representar a função do tendão. A rigidez é afetada pelo treinamento e pela patologia ${ }^{(1)}$.

Outro aspecto importante na análise biomecânica é a performance dos atletas perante os objetivos e funcionalidades de uma partida de alto nível, então, nesta perspectiva, as análises cinéticas e cinemáticas auxiliam o planejamento para melhor rendimento dos futebolistas/clubes.

Nesse sentido, o estudo de Feres, Coelho e Marson ${ }^{(16)}$, foi analisar e comparar os padrões cinemáticos do joelho e as velocidades lineares da coxa e perna no chute com o dorso do pé no futsal em relação aos chutes com diferentes aproveitamentos, sendo estes os que acertaram (CA) ou não acertaram o alvo (CNA). Participaram do estudo 18 universitários homens. Utilizou-se uma câmera filmadora com a frequência de amostragem de $30 \mathrm{~Hz}$. Foram colocados marcadores passivos nos pontos anatômicos do membro inferior dominante. Cada participante realizou 10 chutes. Foram analisados três momentos T1 (fase de preparação), T2 (metade do movimento) e T3 (fase de execução). $\mathrm{Na}$ comparação entre o setor T1-T2 com o T2T3 foram encontradas diferenças significativas $(p<0,001)$, mas na comparação do segmento perna CNA não houve variação na velocidade linear. Assim o padrão de movimento que influenciou no chute foi velocidade linear da perna que não obteve um aumento durante $\mathrm{o}$ segundo setor. Assim podemos concluir que a velocidade linear da perna pode influenciar na direção da bola.

Diante dos estudos analisados podese verificar os benefícios eminentes pela biomecânica ao aprimoramento de técnicas e programas no futebol, podendo melhorar a velocidade dos chutes por meio de observação a padrões cinemáticos ${ }^{(16)}$. A análise biomecânica é fundamental para melhorar desempenho, maior velocidade da bola e menor variabilidade para o membro dominante e também para verificar e corrigir falhas em movimentos que podem estar diretamente relacionadas ao modo como dois atletas não chutam, buscando também otimizar ou executar ${ }^{(9)}$.

As análises biomecânicas por diversos métodos permitem estudar a dinâmica da articulação dos atletas que sofreram lesões e buscam compreender como melhorar sua performance nas partidas.

\section{CONCLUSÃO}

Diante do conteúdo apresentado pode-se concluir que as investigações biomecânicas nos atletas de futebol contribuem significativamente para o prolongamento da carreira esportiva, evitando a interrupção por afecções crônico- 
degenerativas, padronizando procedimentos para

a analisar o movimento esportivo de forma mais

precisa e científica.

\section{REFERÊNCIAS}

1. Cristi-Sánchez I, Danes-Daetz C, Neira A, Ferrada W, Yáñez Díaz R, Silvestre Aguirre R. Patellar and achilles tendon stiffness in elite soccer players assessed using myotonometric measurements. Sports health. 2019;11(2):157-62.

2. Rocha CC, Matias WB, Mascarenhas F. Brasil e Espanha: Gestão das Políticas Públicas Esportivas. REVISTA INTERCONTINENTAL DE GESTÃO DESPORTIVA-RIGD. 2020;10(3):e10020.

3. Silva WM, Bernaldino ES, Fileni $\mathrm{CH}$, Camargo LB, Lima BN, Martins GC, et al. Incidência de lesões muculoesqueléticas em jogadores de futebol profissional no Brasil. Revista CPAQV-Centro de Pesquisas Avançadas em Qualidade de Vida| Vol. 2019;11(3):2.

4. Monteiro JM. Correlação de parâmetros biomecânicos do salto vertical em jogadores de futebol. 2016.

5. Dufour M, Pillu M. Biomecânica Funcional: Membros, Cabeça, Tronco. 1 ed. São Paulo2015.

6. Silva NRMd, Batista TC, Keller K. Análise biomecânica do movimento de gesto derpostivo no futebol: uma revisão de literatura. 2019.

7. Lee JW, Li C, Yung PS, Chan K-M. The reliability and validity of a video-based method for assessing hamstring strength in football players. Journal of Exercise Science \& Fitness. 2017;15(1):18-21.

8. Monajati A, Larumbe-Zabala E, Goss-Sampson M, Naclerio F. Surface electromyography analysis of three squat exercises. Journal of human kinetics. 2019;67(1):73-83.

9. Silva Junior JEPd, Ciccarelli OAC, Pita IMCR, Carvalho CLTd. A importância da análise biomecânica do chute no futebol. Um estudo de caso. Lecturas: Educación física y deportes. 2019;24(251):3.

10. Silvers-Granelli HJ, Bizzini M, Arundale A, Mandelbaum BR, Snyder-Mackler L. Does the FIFA 11+ injury prevention program reduce the incidence of ACL injury in male soccer players? Clinical Orthopaedics and Related Research®. 2017;475(10):2447-55.

11. Arundale AJ, Silvers Granelli HJ, Marmon A, Zarzycki R, Dix C, Snyder Mackler L. Changes in biomechanical knee injury risk factors across two collegiate soccer seasons using the $11+$ prevention program. Scandinavian journal of medicine \& science in sports. 2018;28(12):2592-603.

12. Arundale AJ, Kvist J, Hägglund M, Fältström A. Jump performance in male and female football players. Knee Surgery, Sports Traumatology, Arthroscopy. 2020;28(2):606-13.

13. Krommes K, Petersen J, Nielsen M, Aagaard P,
Hölmich P, Thorborg K. Sprint and jump performance in elite male soccer players following a 10 -week Nordic Hamstring exercise Protocol: a randomised pilot study. BMC research notes. 2017;10(1):1-6.

14. Vassilis S, Yiannis M, Athanasios M, Dimitrios M, Ioannis G, Thomas M. Effect of a 4-week detraining period followed by a 4-week strength program on isokinetic strength in elite youth soccer players. Journal of exercise rehabilitation. 2019;15(1):67.

15. Paiva CPd, Soares Júnior AA. Análise biomecânica da ação do quadril de atletas de futsal com histórico de entorses de tornozelo. Anais da Jornada de Educação Física do Estado de Goiás (ISSN 2675-2050). 2019;1(2):277-81.

16. Feres FC, Coelho DB, Marson RA. Análise cinemática do movimento do chute no futsal com aproveitamentos diferentes. 2017.

OBSERVAÇÃO: Os autores declaram não existir conflitos de interesse de qualquer natureza. 